\title{
Recent Automated Glaucoma Detection Techniques using Color Fundus Images
}

\author{
Prabhjot Kaur, Praveen Kumar Khosla
}

\begin{abstract}
One of the areas in which C-DAC, Mohali is actively engaged, is development of $\mathrm{AI}$ powered fundus imaging system providing insight into several severe eye diseases. Glaucoma, one of the most hazardous ocular disease, continues to affect and burden a large section of our population. Neuropathy of optic nerve cells is the prime cause of glaucoma and is the second leading cause of blindness worldwide. It doesn't manifest itself and is often termed as the silent thief of eye sight. The damage caused by glaucoma is irreversible. Therefore, it is imperative to detect glaucoma at an early stage. The medical literature related to glaucoma indicates that glaucoma detection is a complex process and depends on combination of several parameters. The conventional methods of hand-crafted feature extraction are tedious, time consuming and require human intervention. Even though many such systems have recently shown promising results, but these systems require extensive feature engineering and have limited representation power owing to varied morphology of the optic nerve head. Most of the proposed systems have targeted the parameter cup to disc ratio (CDR) for detection of glaucoma, but that may not be the best approach for building efficient, robust and accurate automated system for glaucoma diagnosis. This paper advocates the use of hybrid approach of manual feature crafting with deep learning. It holds promise of improving the accuracy of glaucoma diagnosis through the automated techniques. It is further proposed that if diagnosis based on CDR remains inconclusive other methods of diagnosis should be adopted to come to a certain conclusion.
\end{abstract}

Index Terms: CDR, CNN, Deep Learning NN, Feature Extraction, Glaucoma, Fundus, ISNT rule, Transfer Learning

\section{INTRODUCTION}

Across the globe, blindness due to Glaucoma is increasing at an alarming rate and is expected to reach 76 million by 2020 and 111.8 million by 2040 [1]. It is a chronic ocular disease categorized by damage to the optic nerve. This is caused mostly by high intraocular pressure which is believed to cause degeneration of ganglion cells of optic nerve. Glaucoma symptoms appear only in the later stage of the disease leading to irreversible vision loss [2]. Since damage caused by glaucoma is irreversible, an early detection and consequent treatment slows down the advancement of the disease. Therefore, a reliable system to detect glaucoma at an early stage is crucial to the treatment. The manual assessment of optic nerve by trained clinicians is, not only time taking but also costly. Therefore, having effective automated methods for detection of glaucoma is the need of the hour.

Revised Manuscript Received on June 15, 2019.

Prabhjot Kaur, Information Technology,

Praveen Kumar Khosla, Executive Director CDAC, Mohali, India.

Automated glaucoma detection is usually performed by extracting structural features through conventional image processing techniques. Optical Coherence Tomography and fundus photography are most widely used imaging techniques in automated glaucoma diagnosis. Recently researchers have also started taking active interest in deep learning techniques for glaucoma detection. In this paper, various procedures for automated glaucoma detection are discussed according to methodology followed, and accuracy obtained. This paper uncovers in the following manner. Section II explains about glaucoma and its important parameters to look for the correct diagnosis of glaucoma. In section III information is given about various Imaging Techniques in use for glaucoma detection. In section IV analysis and comparison of the various computer aided glaucoma detection techniques is made in terms of number of parameters taken into consideration for glaucoma detection, the extent of requirement of preprocessing techniques used, the types of features extracted and the classification techniques used. Section V concludes paper.

\section{GALUCOMA}

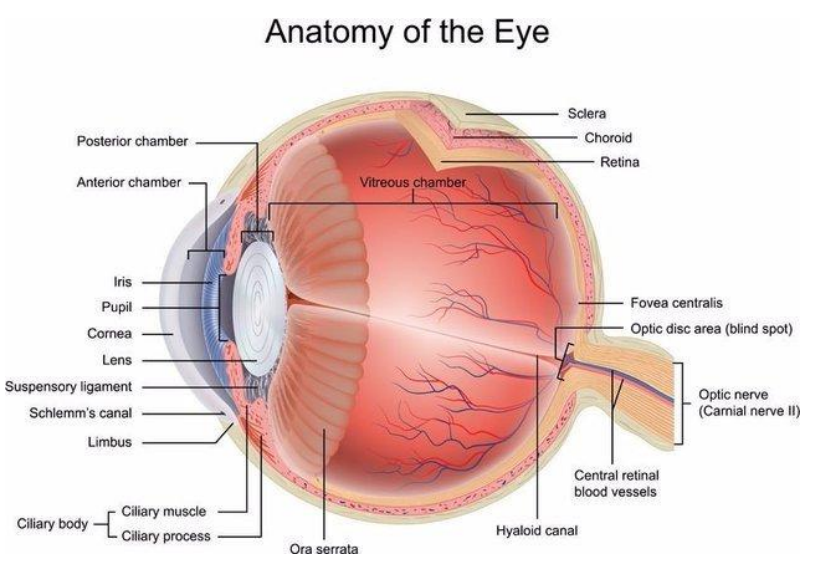

Fig. 1 Anatomy of eye showing optic nerve head

Before creating automated system for glaucoma detection, it is very important to understand about eye and about the procedure for glaucoma diagnosis. Glaucoma is caused by the loss of the axons of retinal ganglion cells which forms the retinal nerve fiber layer at the optic nerve head (ONH) [3].

Figure 1 provides information about eye anatomy and the optic nerve head. Retinal nerve fiber layer (RNFL) forms the prominent part of

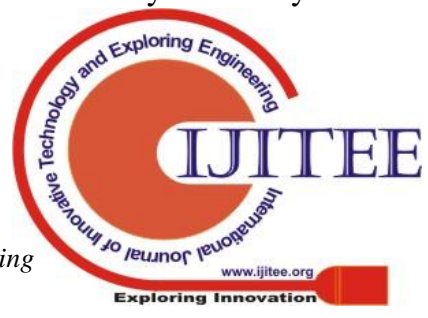


the optic nerve head $(\mathrm{ONH})$. Beneath the RNFL, there are anterior and posterior layers of lamina cribrosa, which consists of laminar dots. The visibility of laminar dots in eye fundus image is more likely to be associated with glaucomatous optic disc. The visibility of laminar dots usually happens because of thinning and deterioration of RNFL. Figure 2 provides information about the same.

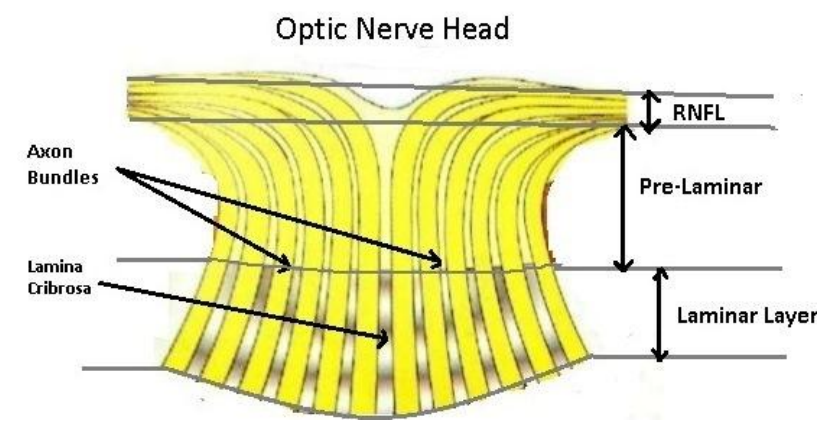

Fig. 2 Cross-Sectional view of Optic Nerve Head

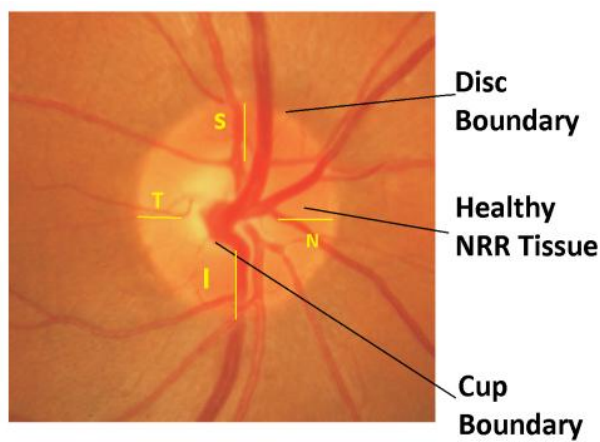

Fig. 3 ROI segmented from CFI showing Healthy Optic Disc showing ISNT rule intact. $\mathrm{I}>=\mathrm{S}>=\mathrm{N}>=\mathrm{T}$

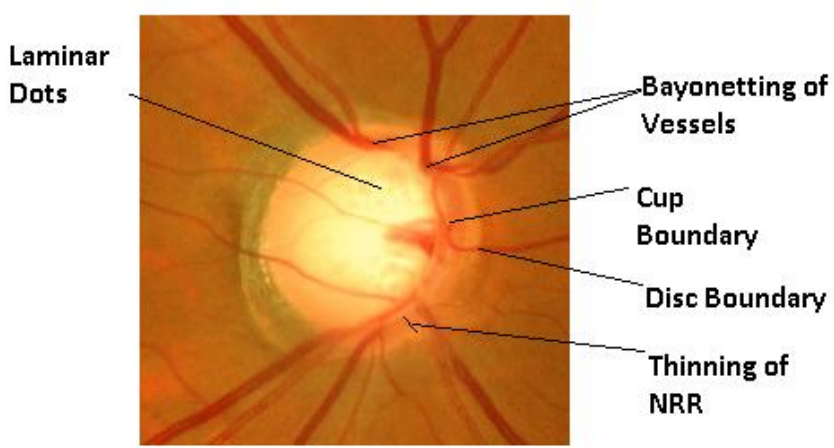

Fig. 4 ROI segmented from CFI depicting Glaucomatous Optic Disc with NRR thinning

Below paragraphs carefully describe the various factors which shall be carefully looked for evaluating glaucoma [4-5, 8] Firstly, determining the size and integrity of the neuroretinal rim (NRR) is of the foremost importance. The Optic disc (OD) is usually oval in shape and has a central cup. The area between external optic disc boundary and the outer optic cup boundary forms NRR [4-5]. Figure 3 shows the healthy optic disc where there is healthy NRR tissue. In general the vertical diameter of OD is greater than

the horizontal disc diameter in an average by $0.11 \mathrm{~mm}$. An OD having vertical diameter less than $1.5 \mathrm{~mm}$ and vertical diameter greater then $2.2 \mathrm{~mm}$, is considered to be typically small and large OD respectively [7-8]. Disc size is an important parameter for assessment of glaucoma. A very small sized OD can be glaucomatous even with the CDR ratio of 0.3 and a large sized OD with the CRD ratio of 0.7 may be non-glaucomatous [9-10].

The normal NRR tissue has regular thickness at all the angles of OD. The sudden or abrupt diminishing of NRR thickness indicates the presence of a notch. Notch is major sign of glaucoma, even though the CDR may be within normal limits [11-13]. ISNT rule is generally true for most of the ODs. ISNT rule states that a normal OD has the heaviest portion of NRR inferiorly and the tinniest portion being the temporal region [14]. Fig. 3 shows a healthy optic disc, having ISNT rule intact. The latest study by Linda Yi-Chieh Poon, et al., proposes to use IST and IS rule instead of ISNT rule, for better glaucoma diagnosis [13].

Other important parameter to look for is the cup to disc ratio (CDR). Higher the CDR value more suspicious is the disc of glaucoma [15], as shown in Fig. 4. The OD shown in figure 4 has high value CDR which is clearly evident from the image. The alteration of the course of blood vessels in OD is good indicator of cup boundary [16].

In uncommonly small sized OD, with low value of CDR may still be a glaucomatous disc. In small sized ODs, it is assumed that CDR value greater than 0.4 makes the disc highly suspicious of glaucoma, and the case should be further thoroughly investigated. A disc having CDR value of 0.8 is judged to be as glaucomatous disc and is major indicator of glaucoma unless proved otherwise [17-18].

Besides the above parameters, other important signs to look for are: optic nerve head hemorrhage, the laminar dots, RNFL defects, peripapillary atrophy in the zone $\beta$, baring of circumlinear vessels, bean potting, bayonetting of vessels and nasalization. Optociliary shunt vessels are also an important indicator of glaucoma [6].

It is also important to mention here that a patient having CDR value in the range of 0.5 to 0.8 and having none of the above symptoms will come under the category of being a glaucoma suspect. In that case, further investigation by the doctor is required to ascertain glaucoma. Futher, Perimetry test of Visual Field and RNFL Optical Coherence Tomography (OCT) test may be performed for making a conclusion [19].

\section{IMAGING TECHNIQUES IN USE FOR GLAUCOMA DETECTION}

Color Fundus Imaging (CFI) is routinely ordered for diagnosing in a wide variety of ophthalmic conditions such as glaucoma, diabetic retinopathy, macular edema and micro aneurysms. Retinal details are easily visualized in stereoscopic fundus photographs as compared with direct examination [20]. CFI uses a low power microscopic camera to record color image of the fundus of eye, including the retina, the optic disc, macula, and posterior pole region [21]. Typical 
photography usually provides an approximately 40 degrees of field of view. It is the cheapest technique compared to other imaging techniques available till date. Fundus photography catches surface view of the retina. Confocal Scanning Laser Ophthalmoscopy, Scanning Laser Polarimetry and Optical Coherence Tomography are the relatively less commonly used techniques as compared to fundus imaging.

Confocal scanning laser ophthalmoscopy (CSLO) use single color laser illumination along with autofluorescence technique to yield detailed view of the ONH. The Heidelberg Retina Tomograph uses laser beam to scan across the fundus. The main advantage of this technique is that it produces in-depth photography [22].

Scanning laser polarimetry (SLP) helps in assessment of RNFL thickness which is an important parameter in glaucoma detection. SLP makes use of concept of light polarization for doing quantitative analysis of thickness assessment. The change of phase of light gives measurement of thickness. It also provides TSNIT (Temporal, Superior, Nasal, Inferior, Temporal) map for evaluation [23].

Optical coherence tomography (OCT) uses light waves to acquire information in depth of the layers of the ONH. In other words it provides 3-D structural, view of the eye layers which is quite helpful in accurately estimating the loss of optic nerve cells [24]. It provides resolution of layers to the order of millimeters. RNFL layer defects and NRR thickness can easily be measured by it which are quite important parameters in glaucoma detection and prediction. The only drawback of OCT is its high cost, therefore it is still not the most used technology in glaucoma detection [25-26].

Though these technologies are quite efficient in capturing structural depth measurements of the fundus, they are quite expensive as compared to CFI. Therefore, it is the most widely used imaging technology in glaucoma detection [27]. To add further, in recent times Smartphone-Fundus Photography is emerging as the new cost effective technique. It captures the image of the fundus with the help of the device camera used with handheld indirect ophthalmoscopy lens. The quality of image is comparable to image taken by fundus cameras [28].

\section{COMPARISON OF VARIOUS AUTOMATED GLAUCOMA DETECTION TECHNIQUES}

A large amount of work for automated glaucoma detection so far is performed on color fundus images as compared to OCT, CSLO or SLP images. Firstly, we will compare systems based in hand crafted feature extraction followed by various deep learning based techniques, followed by transfer learning systems being used for automated glaucoma detection. Table-I. shows comparison chart of various state of the art methods along with their performance. The prominent approaches are explained below in detail.

In [29] U. Raghavendra et al., in 2015, used the energy spectrum based method for glaucoma detection. Initially, optic disc localization is performed by a search window based method. After that Radon transformation (RT) is performed followed by modified census transformation (MCT). GIST descriptor is formed to characterize the image, which generates lots of features. Therefore the features are further normalized by using LSDA. SVM is the classifier used. This system has claimed accuracy of $97.00 \%$.

In [30] S. Maheshwari et al., in 2017 proposed a novel method for glaucoma diagnosis. Initially, EWT is used to image breakdown into various frequency bands. After that correntropy features are obtained from the image. Then feature ranking is done on the value of $t$ value feature selection algorithm. Least squares support vector machine classifier classifies the image between glaucomatous or non-glaucomatous image. This approach boosts of $98.33 \%$ accuracy for 3 fold validation which are quite promising.

In [31], 2017 authors suggested a novel method for glaucoma diagnosis using texton and local configuration pattern based features. Firstly, adaptive histogram equalization is performed, followed by convolution operation of images with various filter banks, resulting in generation of textons. Further, LCP is generated. Local configuration pattern (LCP) refers to distinctive pattern found in image. Feature selection and feature ranking is done by SFFS and statistical t-test respectively along with K-NN classifier. The system boosts of having $95.8 \%$ accuracy.

Julian Zilly et al., [32] in 2016, proposed automatic OC and OD segmentation using a novel method based on CNN. The proposed method used Entropy sampling for selecting sampling points which is claimed to be better than uniform sampling. The selected sampling points are further used to design a learning system of convolutional filters. The extracted OC and OD can be used for CDR calculation which can further be used for glaucoma diagnosis.

Simonthomas, S., et al., [33] in 2014 proposed automated glaucoma diagnosis system by using GLCM and Haralick based texture features. After performing image preprocessing, thirteen Haralick texture features are extracted. Subsequently, KNN classification technique is applied for classifying fundus image from being glaucomatous or healthy. This system claims to have $98 \%$ accuracy in glaucoma detection.

Annu, N., and Judith Justin et al., [34] in 2013 proposed a novel method for glaucoma detection using DWT based textural energy features along with Probabilistic neural network classifier. Wavelet features are generated using five filters specified in [34]. Z-score normalization is performed in the beginning for equalizing the illumination irregularities. This system boosts of achieving 95\% accuracy

In [35] Abhishek Pal et al., in 2018, proposed G-EYENET named autoencoding system. It consists of two model system frame. Initially ROI comprising of OD is obtained from fundus images with the use of modified u-net CNN comprising of binary map. Autoencoder is used for feature learning, following which classification is performed using CNN classifier. HRF, Drishti-GS, RIM ONE v3, datasets are used for training purpose and testing is performed on DRIONS-DB. AUC of 0.923 is achieved with this.

[36] U Raghavendra, et al., in 2017, proposed a 18 layered:- convolution layer(CL), batch normalization layer (BN), Relu(R), MaxPool(MP), CL-2, BN-2, R-2, MP-2, CL-3, BN-3, R-3, MP-3,CL-4, BN-4, R-4, MP-4, fully connected layer, and softmax layer, CNN architecture for glaucoma diagnosis using 
CFIs. In preprocessing phase all the fundus images are resized to 64X64 sized images. Authors experimented with different learning rates of $0.1,0.01,0.001$, and 0.0001 to find various parameters like accuracy, sensitivity, specificity and PPV. 0.001 is found to be the optimal learning rate. Accuracy of $98.13 \%$ is achieved. The dataset used is of 1426 fundus images as accuracy of CNN is highly dependent on the variability and scope of the training dataset used.

In 2015, [37] Xiangyu Chen et al., proposed 6 layered $\mathrm{CNN}$. Over fitting problem is also taken care of by using response-normalization and with pooling layers. The system also used dropout and data augmentation strategies to improve performance. ORIGA and SCES datasets were used. (AUC) of the receiver operating characteristic curve in glaucoma detection is 0.831 from ORIGA dataset and 0.887 from SCES dataset.

In [38] Juan J. Gómez-Valverde et al., in 2019 made a system for glaucoma detection using the concept of transfer learning. In preprocessing phase localization of optic disc is performed with the help of morphological operations and the localized image is further scaled to $256 \times 256$ pixels. VGG-19 net is used for transfer learning. Comparison of performance is made between simple CNN based approach and transfer learning based approach. Transfer learning scheme with VGG19 clearly outperforms the other approach achieving an AUC of 0.94.Total of 2313 images are used (RIM-ONE 49,DRISHTI-GS 50, and 1 Private Data set).

In [39] Alan Carlos de Moura Lima, et al., in 2018, a comparison study is made between various CNNs. A number of CNNs comprising Visual Geometry Group architecture version 16 and 19, Residual Neural Network architecture version 50 and Inception CNNs are used for the study. Previously trained weights of image net challenge are used with all. A large number of features were extracted by each of CNN architecture per image forming five datasets for each image. Each of the above was tested with different prominent classifiers. CNN ResNet50 got the best result of accuracy of $90 \%$ with the $\mathrm{r} 2$ version of RIM-ONE and Logistic Regression classifier.

Annan Li et al., [40] in 2018 proposed an approach based on combining multiple features extracted by different CNNs. First OD is extracted from CFI using deformable shape model. From the OD, ROI is extracted and normalized to $256 \times 256$ pixel size. Features are extracted from following CNNs: AlexNet, VGG-16, VGG-19, GoogLeNet, ResNet-50, ResNet-152. All the features extracted from above are concatenated to obtain a single feature vector of 19,456 number of extracted features. In the last step, SVM classifier is applied for classification between healthy or glaucomatous eye. The AUC achieved is 0.838

Yidong Chai et al., [41] in 2018, proposed a MBNN based novel approach for glaucoma diagnosis. This approach tried combining various types of $\mathrm{CNN}$ for extracting various parameters important for glaucoma diagnosis. The extracted knowledge parameters for glaucoma diagnosis includes CDR, RNFLD. The Accuracy achieved is of $91.5 \%$.

Hemanth et al., [42] in 2018 proposed Modified Hopfield Neural Network technique to diagnose Diabetic from the Retinal Images. Mittal et al., [43] in 2019 proposed efficient edge detections when images are to be analyzed, further they applied Deep learning techniques to analysis brain tumor [44-45]. The proposed innovative salient feature extraction techniques can be applied to glaucoma detection as well.

Table I. Prominent Nobel Methods for Glaucoma Detection- comparison

Method / Feature Extracted /
Technique used

[29] 2018, non-parametric spatial envelope energy spectrum based method, Radon Transformaton + Modified Census Transformation + GIST descriptor + Locality Sensitive Discriminant Analysis LSDA

\begin{tabular}{|c|c} 
Glaucoma \\
Detection \\
Performance \\
Analysis
\end{tabular}

[30] 2017, Empirical Wavelet Transformation, Correntropy based Feature extraction, Feature selection and ranking by t-value algorithm, Least Squares Support Vector Machine as classifier



Private Dataset Acc: $97.00 \%$, Public Dataset Acc: $93.62 \%$

[31] 2017, Texton and Local Configuration Pattern (LCP) based features, SFFS feature selection, statistical t-test feature ranking, K-NN Classification

[32] 2016, OC and OD segmentation using Entropy sampling and Ensemble learning

(learning

[33] 2014, GLCM + 13 Haralick texture features and $\mathrm{KNN}$ as classifier

\begin{tabular}{|l|l|}
\hline $\begin{array}{l}\text { [34] 2013, DWT based Textural } \\
\text { Energy Features, Probabilistic Neural } \\
\text { Network (PNN) Classifier }\end{array}$ & Acc: $95 \%$ \\
\hline $\begin{array}{l}\text { [35] 2018, G-EYENET convolutional } \\
\text { autoencoding classifier framework, } \\
\text { and traditional CNN as classifier, } \\
\text { HRF, DRISHTI-GS,RIM ONE v3 } \\
\text { dataset for training, DRIOND-DB for } \\
\text { testing }\end{array}$ & AUC: 0.923 \\
\hline
\end{tabular}

Acc: $98 \%$

Segmentation only

Acc: $95.8 \%$ Exploring Innovation 
[36] 2017, 18 layered CNN architecture, 1426 Fundus Images , 589 normal and 837 glaucomatous

Acc: $98.13 \%$

[37] 2015, 6 layered CNN, 2 convolution

ORIGA AUC: layers, 4 fully connected layers. Dataset:

0.831, SCES ORIGA and SCES

AUC: 0.887

[38] 2019, Transfer Learning. OD as ROI extraction, image scaling, VGG-19 used

AUC: 0.94 for transfer learning

[39] 2018, Features are extracted from CNN ResNet-50 with pre-trained weights from ImageNet challenge , Logistic Regression Classifier, RIM-ONE r2 as dataset

[40] 2018 Features extracted from AlexNet, VGG-16, VGG-19, GoogLeNet, ResNet-50, ResNet-152. Total number of features extracted: 19,456, SVM Classifier

[41] 2018, Multi-branch neural network (MBNN), use of Domain Knowledge: CDR,RNFLD,PPA and Patient data

Acc: $91.5 \%$

\section{CONCLUSION}

There are several systems having conventional hand crafted feature extraction algorithms based on EWT, correntropy feature extraction, DWT, HOS, Gabor Transformation, Wavelet energy features, Haralick, entropy based features showing promising results and seem to have higher level of accuracy as compared to Deep Learning based methods. At the same time, due to recent availability of high performing GPUs, Deep Learning techniques have become more powerful. The biggest advantage of deep learning is, automatic incremental high level feature learning. CNNs have very good capability in automatic feature extraction and learning, so they have the great ability in representing non-linear functions also. The main constraint in deep learning is to have large dataset, depicting sufficiently different types of glaucomatous fundus images. Awakening of the medical community to record fundus images has increased the availability of a large number of fundus images. It is becoming possible to design a highly, efficient and accurate deep learning based glaucoma diagnosis system. Various combinations of deep learning neural networks, transfer learning neural networks with hybrid feature extraction and extensive training can be adopted for improved results.

\section{REFERENCES}

1. Y. C. Tham, X. Li, T. Y. Wong, H. A. Quigley, T. Aung, and C. Y. Cheng, "Global prevalence of glaucoma and projections of glaucoma burden through 2040: A systematic review and meta-analysis," J. Ophthalmology, vol. 121, no. 11, pp. 2081-2090, 2014.

2. American Optometric Association, "Glaucoma" https://www.aoa.org/patients-and-public/eye-and-vision-problems/glos sary-of-eye-and-vision-conditions/glaucoma, Access Date: 1 May 2019

3. Downs, J. C., Roberts, M. D., \& BURGOYNE, C. F. (2008). "Mechanical Environment of the Optic Nerve Head in Glaucoma". Optometry and Vision Science, 85(6), E425-E435.

4. Jonas JB, Budde WM, "Diagnosis and Pathogenesis of Glaucomatous Optic Neuropathy: Morphological Aspects", Prog Retin Eye Res, 2000; $19: 1-40$

5. American Academy of Opthalmology, "Clinical Evaluation of the Optic Nerve Head", https://www.aao.org/bcscsnippetdetail.aspx?id=ee28fa7a-e1f7-4495-9 9a4-7828a800fcd2, Access Date: 3 May 2019

6. Naida Jakirlic ,'Optic Nerve Evaluation in Glaucoma', California Optometric Association", 2016

7. Downs, J. C., Roberts, M. D., \& BURGOYNE, C. F. (2008) "Mechanical Environment of the Optic Nerve Head in Glaucoma". Optometry and Vision Science, 85(6), E425-E435.

8. Susanna Jr R, Medeiros FA, "Ophthalmoscopic aspects of the optic nerve in glaucoma: Normal optic disc", Rio de Jenerio: Cultura Médica, 2006, 1-12.

9. Hoffmann EM, Zangwill LM, Crowston JG, Weinreb RN. “Optic disk size and glaucoma”. Surv Ophthalmol.2007;52(1):32-49.doi:10.1016/j.survophthal.2006.10.00 2.

10. Peter E. Libre, MD, Norwalk, Conn. "Revisiting the Importance of Disc Size, Review of Ophthalmology", 2004.

11. Spaeth GL, Lopes JF, Junk AK, et al., "Systems for staging the amount of optic nerve damage in glaucoma: A critical review and new material", Surv Ophthalmol, 2006; 51:293-315.

12. Fingeret M, Medeiros FA, Susanna Jr R, Weinreb RN, "Five rules to evaluate the optic disc and retinal nerve fiber layer for glaucoma", Optometry, 2005;76:661-8.

13. Linda Yi-Chieh Poon, David Solá-Del Valle, Angela V. Turalba, Iryna A. Falkenstein, Michael Horsley, Julie H. Kim, Brian J. Song, Hana L. Takusagawa, Kaidi Wang, Teresa C. Chen, "The ISNT Rule: How Often Does It Apply to Disc Photos and Retinal Nerve Fiber Layer Measurements in the Normal Population”, Am J Ophthalmol, 2018.

14. W. Ruengkitpinyo ; W. Kongprawechnon ; T. Kondo ; P. Bunnun ; H Kaneko , "Glaucoma screening using rim width based on ISNT rule", IEEE 2015 6th International Conference of Information and Communication Technology for Embedded Systems (IC-ICTES).

15. Andrew J. Tatham; Robert N. Weinreb; Linda M. Zangwill; Jeffrey M. Liebmann; Christopher A. Girkin; Felipe A. Medeiros,'The Relationship Between Cup-to-Disc Ratio and Estimated Number of Retinal Ganglion Cells", Investigative ophthalmology and visual science, 2013.

16. Jonas JB, Gusek GC, Naumann GO, "Optic disc, cup and neuroretinal rim size, configuration and correlations in normal eyes", Invest Ophthalmol VisSci, 1988;29:1151-8.

17. Hayreh SS, "Ischemic Optic Neuropathies", Berlin Heidelberg: Springer-Verlag, 2011.

18. Bourne RR, "The optic nerve head in glaucoma", Community Eye Health, 2006;19:44-5.

19. Mandeep Singh, Mooninder Singh, JiwanpreetKaurVirk, "Glaucoma Detection Techniques: A Review", International Journal of Computer Science \& Communication (ISSN: 0973-7391), Vol 6 • Number 2 April - Sep 2015 pp. 66-76.

20. https://www.sciencedirect.com/topics/ medicine-and-dentistry/fundus-photography, access date : 1 May 2019

21. Abràmoff $\mathrm{MD}$, Garvin $\mathrm{MK}$, Sonka $\mathrm{M}$, "Retinal imaging and image analysis", PubMed, 2010

22. C Alexandrescu, AM Dascalu, A Panca, A Sescioreanu, C Mitulescu, R Ciuluvica, L Voinea, C Celea, "Confocal scanning laser ophthalmoscopy in glaucoma diagnosis and management", $\mathrm{J}$ Med Life. 2010 Aug 15; 3(3),pp: 229-234. 
23. Weinreb RN, et al., "Scanning laser polarimetry to measure the nerve fiber layer of normal and glaucomatous eyes". Am J Ophthalmology. 1995;119(5),pp::627-36.

24. Huang D, Swanson EA, Lin CP, Schuman JS, Stinson WG, Chang W, Hee MR, Flotte T, Gregory K, Puliafito CA, "Optical coherence tomography”, Science. 1991 Nov 22; 254(5035):1178-81.

25. James G Fujimoto, Costas Pitris, Stephen A Boppart, "Optical Coherence Tomography: An Emerging Technology for Biomedical Imaging and Optical Biopsy", PMC, 2000.

26. Tehmina Khalil ; Muhammad Usman Akram ; Samina Khalid ; Amina Jameel, “An overview of automated glaucoma detection”, IEEE 2017 Computing Conference.

27. Michael Abràmoff, Christine N. Kay, Chapter 6 Image Processing, Book: Retina, Elsevier, 2013, doi 10.1016/B978-1-4557-0737-9.00006-0.

28. Hossein Nazari Khanamiri, Austin Nakatsuka, and Jaafar El-Annan, "Smartphone Fundus Photography", PubMed, 2017

29. U. Raghavendra, Sulatha V. Bhandary, Anjan Gudigar, U. Rajendra Acharya, "Novel expert system for glaucoma identification using non-parametric spatial envelope energy spectrum with fundus images", Bio cybernetics and Biomedical Engineering, Volume 38, Issue 1, 2018, pp:170-180.

30. S. Maheshwari, R. B. Pachori, and U. R. Acharya, "Automated Diagnosis of Glaucoma Using Empirical Wavelet Transform and Correntropy Features Extracted From Fundus Images," IEEE J. Biomed. Health Inform. 21(3), (2017) pp: 803-813.

31. U Rajendra Acharya, Shreya Bat, Joel EW Koh, Sulatha V Bhandary, Hojjat Adeli, A novel algorithm, to detect glaucoma risk using texton and local configuration pattern features extracted from fundus images, Computers in Biology and Medicine, vol. 88 (2017),pp: 72-83.

32. Julian Zilly, Joachim M. Buhmann, Dwarikanath Mahapatra, "Glaucoma detection using entropy sampling and ensemble learning for automatic optic cup and disc segmentation", Computerized Medical Imaging and Graphics, Elsevier, Volume 55, January 2017, pp: $28-41$.

33. Simonthomas, S., N. Thulasi, and P. Asharaf. "Automated diagnosis of glaucoma using Haralick texture features." In Information Communication and Embedded Systems (ICICES), 2014 International Conference on, pp. 1-6. IEEE, 2014.

34. Annu, N., and Judith Justin. "Automated classification of glaucoma images by wavelet energy features." International Journal of Engineering and Technology 5, no. 2 (2013),pp: 1716-1721.

35. Abhishek Pal, Manav Rajiv Moorthy, A. Shahina , "G-EYENET: a convolutional autoencoding classifier framework for the detection of glaucoma from retinal fundus images", 25th IEEE International Conference on Image Processing (ICIP), 2018.

36. U Raghavendra, Hamido Fujita, Sulatha V Bhandary, Anjan Gudigar, Jen Hong Tan, U Rajendra Acharya, "Deep Convolution Neural Network for Accurate Diagnosis of Glaucoma Using Digital Fundus Images", Computerized Medical Imaging and Graphics, ScienceDirect, Elsevier, Volume 55, January 2017, pp: 28-41.

37. Xiangyu Chen, Yanwu Xu, Damon Wing Kee Wong, Tien Yin Wong, Jiang Liu, "Glaucoma Detection based on Deep Convolutional Neural Network", 37th Annual International Conference of the IEEE Engineering in Medicine and Biology Society (EMBC), 2015.

38. Juan J. Gómez-Valverde et al., "Automatic glaucoma classification using color fundus images based on convolutional neural networks and transfer learning", Biomedical Optics Express, Vol. 10, No. $2 \mid 1$ Feb 2019.

39. Alan Carlos de Moura Lima, Lucas Bezerra Maia, Roberto Matheus Pinheiro Pereira, Geraldo Braz J'unior, Joao Dallyson Sousa de Almeida, Anselmo Cardoso de Paiva, "Glaucoma Diagnosis over Eye Fundus Image through Deep Features", 25th International Conference on Systems, Signals and Image Processing (IWSSIP), 2018.

40. Annan Li, Yunhong Wang, Jun Cheng, Jiang Liu, "Combining Multiple Deep Features for Glaucoma Classification”, 2018 IEEE International Conference on Acoustics, Speech and Signal Processing (ICASSP).

41. Yidong Chai, Hongyan Liu, JieXu, "Glaucoma diagnosis based on both hidden features and domain knowledge through deep learning models", Knowledge-Based Systems, ScienceDirect, Volume 161, 1 December 2018, 147-156.

42. D. J Hemanth, J. Anitha, L Hoang Son, M Mittal "Diabetic Retinopathy Diagnosis from Retinal Images using Modified Hopfield Neural Network", Journal of Medical Systems, 42(2018),pp:247.

43. M Mittal, A Verma, I Kaur, B Kaur, M Sharma, L M Goyal, S Roy \& T-H Kim, “An Efficient Edge Detection Approach to Provide Better
Edge Connectivity for Image Analysis", IEEE Access, Vol. 7(2019), pp:33240-33255.

44. Kaur B., Sharma M., Mittal M., Verma A., Goyal L. M., Hemanth D. J, "An improved salient object detection algorithm combining background and foreground connectivity for brain image analysis", Computers and Electrical Engineering, vol. 71(2018), pp:692-703.

45. M. Mittal, L M Goyal, S Kaur, I Kaur, A Verma, D. J Hemanth, "Deep learning based enhanced tumor segmentation approach for MR brain images", Applied Soft Computing, Vol 78 (2019), pp:346-354.

\section{AUTHORS' PROFILE}

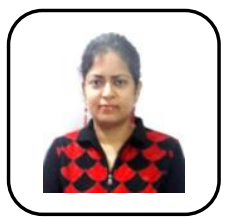

Ms. Prabhjot Kaur is M.Tech. Research student in the department of Information Technology at CDAC Mohali, India. Her interests are in the field of artificial intelligence and machine learning.

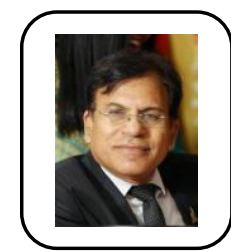

Dr P K Khosla is Executive Director of C-DAC, Mohali and is recipient of "Scientist of the Year Award-2015" of DRDO. He has several research papers and book chapters to his credit. 